\title{
A characteristic length scale causes anomalous size effects and boundary programmability in mechanical metamaterials
}

\author{
Corentin Coulais $^{1,2,3 \star}$, Chris Kettenis ${ }^{2}$ and Martin van Hecke ${ }^{1,2}$
}

\begin{abstract}
The architecture of mechanical metamaterials is designed to harness geometry ${ }^{1-6}$, nonlinearity ${ }^{7-11}$ and topology ${ }^{11-15}$ to obtain advanced functionalities such as shape morphing ${ }^{7,9,16-21}$, programmability $^{18,22,23}$ and one-way propagation ${ }^{11,13,14}$. Although a purely geometric framework successfully captures the physics of small systems under idealized conditions, large systems or heterogeneous driving conditions remain essentially unexplored. Here we uncover strong anomalies in the mechanics of a broad class of metamaterials, such as auxetics ${ }^{2,5,24}$, shape changers $^{16-21}$ or topological insulators ${ }^{11-13,15}$; a non-monotonic variation of their stiffness with system size, and the ability of textured boundaries to completely alter their properties. These striking features stem from the competition between rotationbased deformations-relevant for small systems-and ordinary elasticity, and are controlled by a characteristic length scale which is entirely tunable by the architectural details. Our study provides new vistas for designing, controlling and programming the mechanics of metamaterials.
\end{abstract}

A central strategy for the design of metamaterials leverages the notion of a mechanism, which is a collection of rigid elements linked by completely flexible hinges, designed to allow for a collective, free rotational motion of the elements. Mechanismbased metamaterials borrow the geometric design of mechanisms, but instead of hinges feature flexible parts which connect stiffer elements $^{2,3,5,9,11-13,15-18,22,23,25,26}$. The tacit assumption is then that the low-energy deformations of such metamaterials are similar to the free motion of the underlying mechanism, and the ability to control deformations by geometric design is the foundation for the unusual mechanics of a wide variety of mechanical metamaterials. Such mechanism-based metamaterials have mostly been studied for small systems and for homogeneous loads, where the response indeed closely follows that of the underlying mechanism. However, the physics of large systems, or for inhomogeneous boundary conditions, remains largely unexplored.

We first illustrate that deformations of mechanism-based metamaterials deviate from those of their underlying mechanism under inhomogeneous forcing. Specifically, we consider point forcing of a paradigmatic metamaterial (Fig. 1a), which is based on a mechanism consisting of counter-rotating hinged squares (Fig. 1b) $)^{2,7,9,10,23,24}$. Whereas the local deformations mimic that of the underlying mechanism, at larger scales, we observe that the counter-rotations slowly decay away from the boundary (Fig. 1c). This indicates elastic distortions of the underlying rotating square mechanism, where no such decay can occur. In this example, two-dimensional (2D) effects complicate the physics, and we therefore focus on quasi-1D meta-chains, consisting of $2 \times N$ square elements of diagonal $L$ linked at their tips (Fig. 2a,b); whenever convenient, we will express lengths in units of $L$. We measure the linear response of these samples by forcing the outer horizontal joints. Surprisingly, both experiments and finite element (FEM) simulations show an exponential decay of the mechanism-like rotations away from the boundary when the meta-chain is stretched or compressed (Fig. 2c). This spatial decay defines a novel characteristic length $n^{*}$ (Fig. 2c inset), and suggests that elastic distortions of the underlying mechanism are a general feature of mechanismbased metamaterials.

A first striking consequence of these distortions emerges when probing the effective stiffness of mechanism-based metamaterials as a function of system size. Whereas for elastic continua the effective spring constant or stiffness is inversely proportional to the linear size $^{27}$, experiments and FEM simulations of meta-chains reveal remarkable deviations from this behaviour. For small systems we find that the stiffness $k_{\mathrm{o}}$ for odd $N$ is much larger than the stiffness $k_{\mathrm{e}}$ for even $N$. Moreover, whereas $k_{\mathrm{o}}$ decays monotonously with $N, k_{\mathrm{e}}$ initially increases with $N$. Eventually the stiffness $k_{\mathrm{e}}$ peaks at length $n_{\mathrm{p}}$, and for larger $N, k_{\mathrm{e}}$ approaches $k_{\mathrm{o}}$ and both decay with system size (Fig. 2d). This anomalous size dependence is a robust featurewe have numerically determined the size-dependent stiffness for the 2D metamaterial shown in Fig. 1, as well as a 3D generalization of these ${ }^{18}$, and find that these exhibit a similar peak in stiffness (see Supplementary Fig. 2).

The stiffness anomaly reflects the hybrid nature of mechanismbased metamaterials, as can be seen by comparing two simple models. Whereas a chain consisting of $N$ unit springs of stiffness $\kappa$ in series has a global spring constant $k$ that is inversely proportional to the system size $N: k=\kappa / N$, the stiffness of a rotating squares chain where all hinges are dressed by torsional springs of stiffness $C_{\mathrm{b}}$ (refs $11,16,23$ ) does not decay with $N$. Specifically, for even $N$, the local rotation $\Omega$ and globally applied deformation $u$ are of the same order, and the spring constant $k_{\mathrm{e}} \sim N$-longer chains are thus stiffer in this model. For odd $N$, the counter-rotating motions cancel in leading order, so that $\Omega \ggg u$ and $k_{\mathrm{o}}$ diverges (see Supplementary Information, 'Model B'). Hence, whereas the total deformation in a spring chain is evenly distributed over all elastic elements, such homogeneity breaks down for mechanisms, precisely because of the counter-rotations. The response of flexible, mechanism-based metamaterials hybridizes pure mechanism-like and homogeneous elastic deformations, leading to a crossover from

\footnotetext{
${ }^{1}$ AMOLF, Science Park 104, 1098 XG Amsterdam, the Netherlands. ${ }^{2}$ Huygens-Kamerlingh Onnes Lab, Universiteit Leiden, PO Box 9504,2300 RA Leiden, the Netherlands. ${ }^{3}$ Van der Waals-Zeeman Institute, Institute of Physics, Universiteit van Amsterdam, Science Park 904,1098 XH Amsterdam, the Netherlands. *e-mail: coulais@uva.nl
} 
$\mathbf{a}$

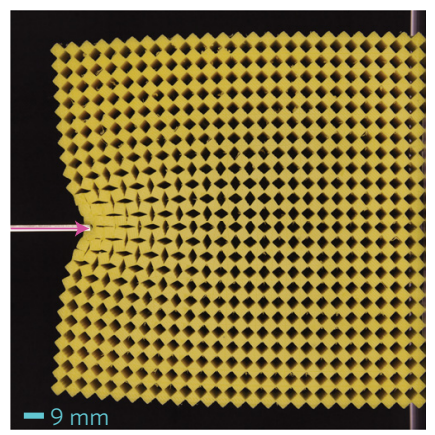

b
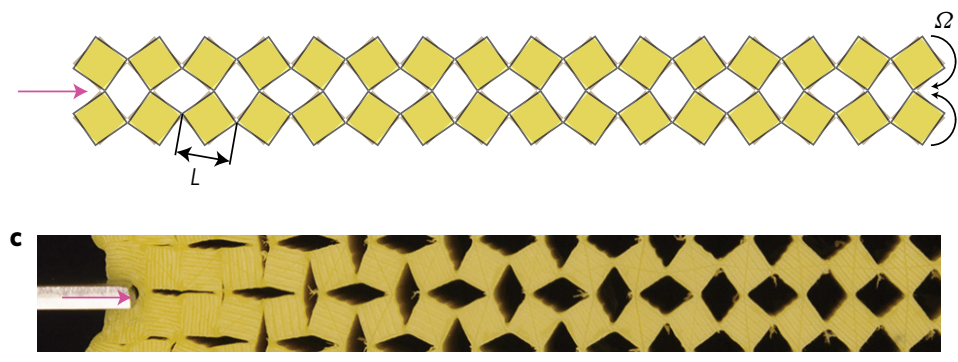

Figure 1 | Mechanism-based metamaterials. a, A paradigmatic example of a mechanism-based metamaterial consisting of rubber slab patterned with a regular array of holes $2,7,9,10,23,24$. Point forcing concentrated near the slender link between two square elements yields a characteristic diamond-platter pattern near the tip and more smooth deformations further away (scale bar, $9 \mathrm{~mm}$ ). $\mathbf{b}$, The rotating squares mechanism ${ }^{2}$ consists of counter-rotating, hinged rigid squares with diagonal $L$ and underlies the design of the metamaterial shown in $\mathbf{a}$. The deformation from the symmetric state can be specified by a single angle $\Omega$.c. A zoom-in reveals that the deformation field of the mechanism-based metamaterial is highly textured, with the rotation $\Omega$ slowly decaying away from the boundary-here, $L=6.4 \mathrm{~mm}$.

a

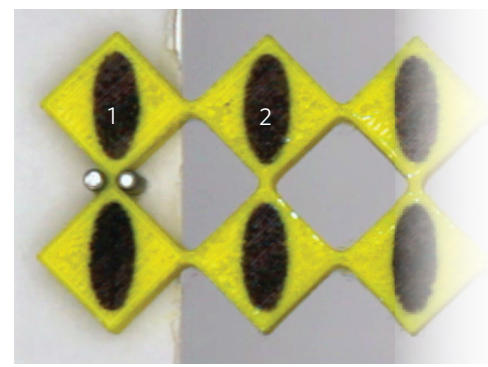

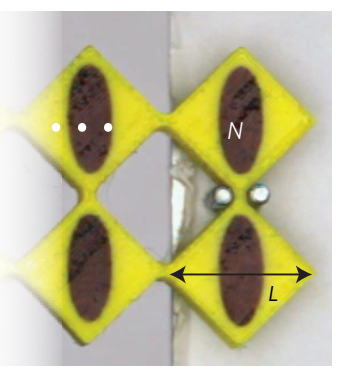

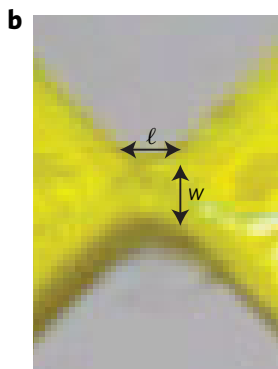

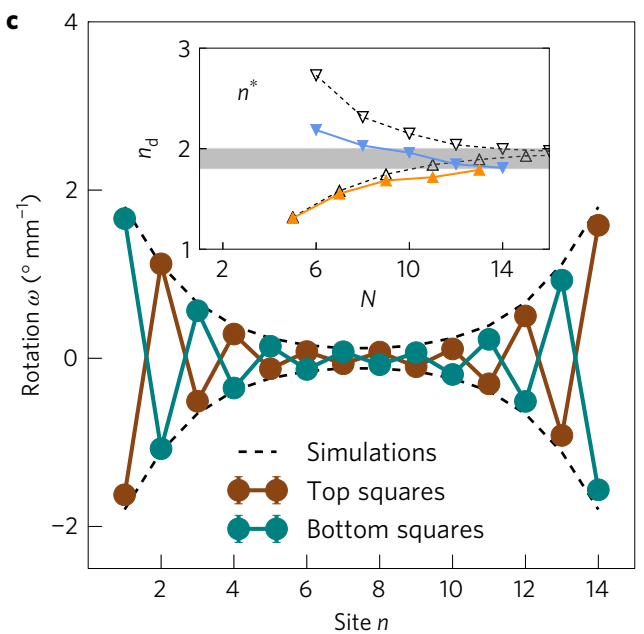

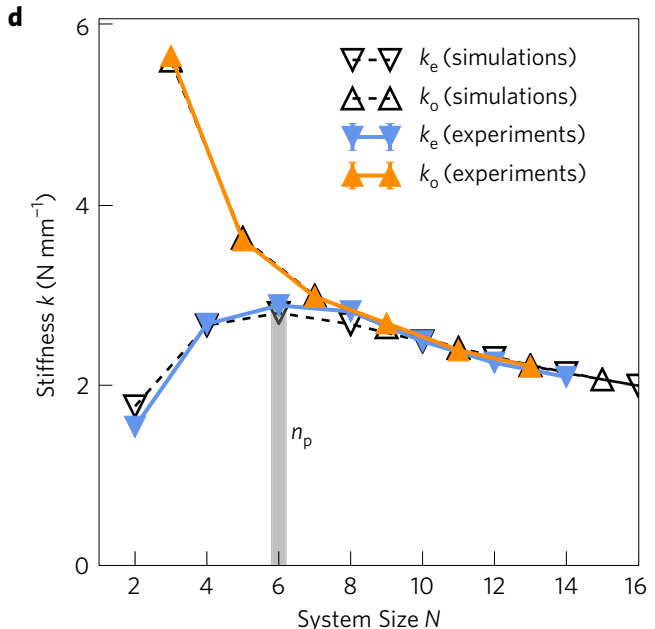

Figure 2 | Anomalies in the stiffness and deformations of meta-chains. a, 3D printed meta-chain of length $N$, thickness $H=7.5 \mathrm{~mm}$ and square diagonal $L=17 \mathrm{~mm}$; the black ellipses are used for tracking positions and rotations. $\mathbf{b}$, Hinge geometry defined by $\ell$ and $w$. c, Rotation field for a meta-chain of length $N=14$-the rotation rate $\omega(n)$ is the proportionality coefficient between the rotations of the square elements and the deformation $\delta$ (see Methods and Supplementary Fig. 1). Maroon (blue) symbols denote the data obtained from the upper (lower) squares. Inset: the decay length converges to a well defined value $n^{*}=1.9 \pm 0.1$ in the limit of large system size. Coloured symbols denote experiments for odd ( $k_{0}$; orange) and even ( $k_{e}$; blue) meta-chains, and dashed curves denote FEM simulations, for $\ell=1.7 \mathrm{~mm}, w=1.7 \mathrm{~mm}$. d, Stiffness as function of $N$ (see Methods). The stiffness $k_{\mathrm{e}}$ peaks at $n_{\mathrm{p}}=6.0 \pm 0.1$.

a mechanism-dominated, inhomogeneous regime for small systems to a homogeneous elastic regime for larger system sizes.

Both $n^{*}$ and $n_{\mathrm{p}}$ reveal this crossover, but we note that their values differ. To understand what sets these values and untangle their relation, we consider a hybrid dressed mechanism where the hinges are subject to bending, stretching and shear, with stiffnesses $C_{\mathrm{b}}, k_{\mathrm{j}}$ and $C_{s}$, respectively (see Supplementary Information, 'Model BSS'). Stretching and shear introduce deformations that compete with the purely counter-rotating mode of the underlying mechanism. The equations that govern mechanical equilibrium are controlled by the dimensionless ratios (see Supplementary Information):

$$
\alpha=\left(1+\frac{L}{\ell}\right)^{2} \frac{C_{\mathrm{s}}}{4 C_{\mathrm{b}}} ; \quad \beta=\frac{k_{\mathrm{j}} L^{2}}{4 C_{\mathrm{b}}}
$$

which tune the relative elastic penalties of mechanism-preserving and mechanism-distorting deformations. The purely torsional model corresponds to the limit where both the stretching and 

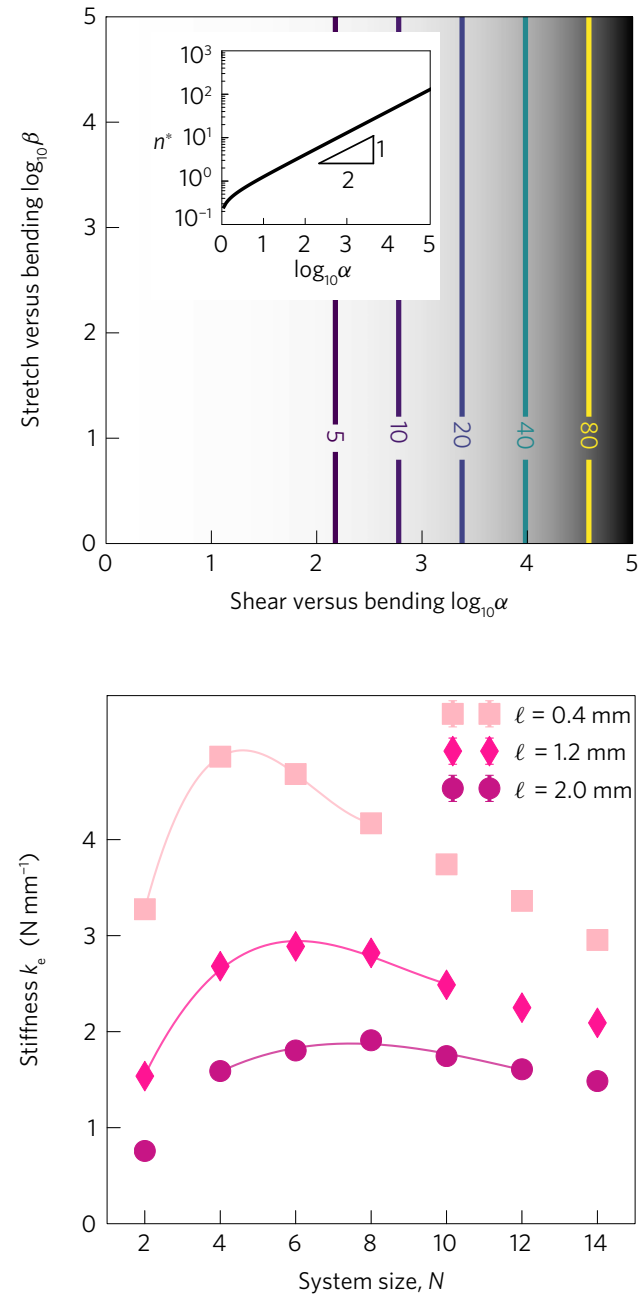
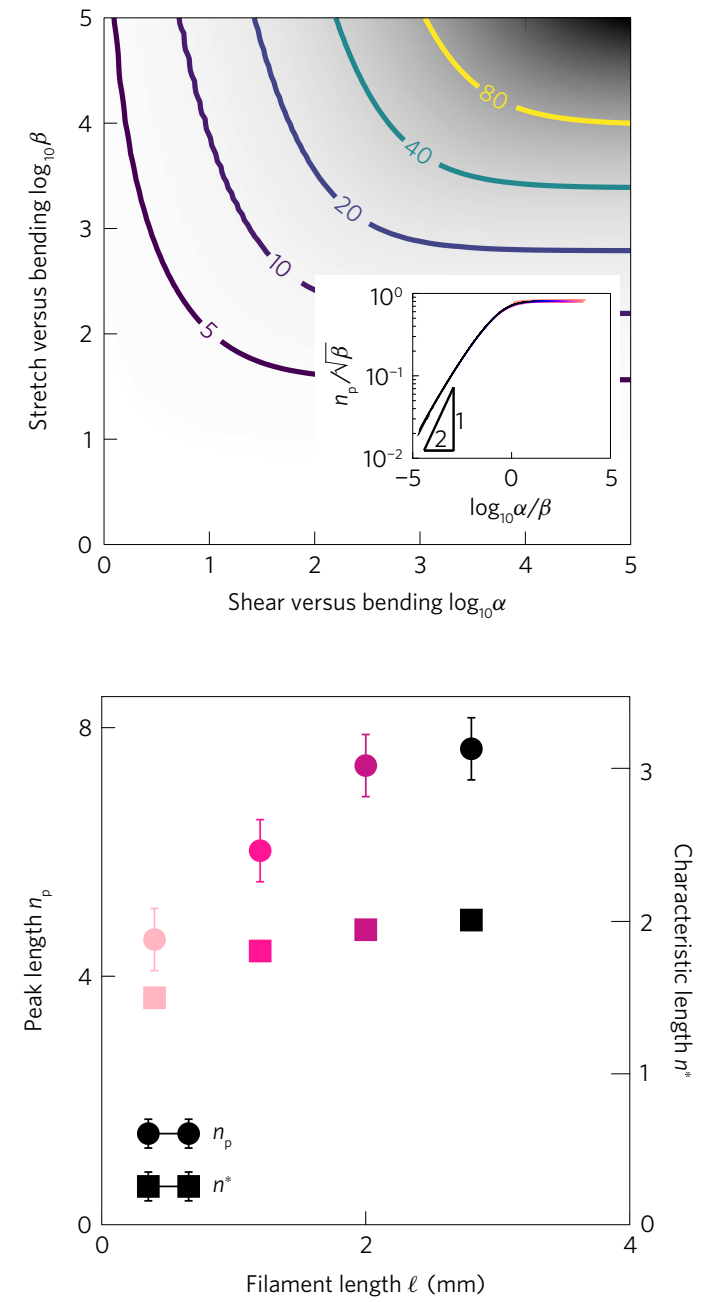

Figure 3 | Characteristic scales. a,b, Contour plots of $n^{*}(\mathbf{a})$ and $n_{\mathrm{p}}$ (b) versus the shear-to-bending ratio $\alpha$ and the stretch to bending ratio $\beta$ computed for the hybrid mechanism (see Methods). Insets show the characteristic length $n^{*}$ scales as the square root of $\alpha$ (a) and the peak length scale $n_{\mathrm{p}}$ collapses onto a master curve when plotted as $n_{p} / \sqrt{\beta}$ versus $\alpha / \beta$ (b). c, Stiffness versus system size in experiments with different filament length $\ell$. We fit a cubic function (continuous curves) to the data near the peak of $k_{\mathrm{e}}$, allowing us to estimate $n_{\mathrm{p}}$ to within \pm 0.5 , which corresponds to the average $95 \%$ interval of confidence of the fits. $\mathbf{d}$, Corresponding location of the length scales $n_{p}$ (disks) and $n^{*}$ (squares) versus filament length $\ell$.

shear stiffnesses are much larger than the bending stiffness (that is, $\alpha, \beta \rightarrow \infty)$. We have checked that solutions to this model for appropriate values of $\alpha$ and $\beta$ show excellent agreement with the experimental results (see Methods and Supplementary Figs 3 and 4): dressing the mechanism with elastic hinge interactions is an effective approach to describe mechanism-based metamaterials.

The competition between mechanism-preserving and mechanism-distorting deformations controls the characteristic length scale. To show this, we vary the control parameters $\alpha$ and $\beta$, and determine $n^{*}$ and $n_{\mathrm{p}}$. When mechanism-like deformations are energetically cheap (large $\alpha, \beta$ ), both $n^{*}$ and $n_{\mathrm{p}}$ diverge, whereas when rotations are energetically expensive (small $\alpha, \beta)$, the lengths $n^{*}$ and $n_{\mathrm{p}}$ become small (Fig. 3a,b). Experimentally, we can leverage this connection to vary and control the length scale, as the relative costs of the mechanism-preserving and mechanism-distorting deformations are controlled by the hinge geometry. To demonstrate this, we have varied the experimental hinge length $\ell$ to push the stiffness ratios $\alpha$ and $\beta$ up, and we find that increasing $\ell$ indeed leads to an increase of both $n^{*}$ and $n_{\mathrm{p}}$ (Fig. $3 \mathrm{c}, \mathrm{d}$ ).

Strikingly, $n^{*}$ is independent of $\beta$ whereas $n_{\mathrm{p}}$ depends on both $\alpha$ and $\beta$, and as we will show below, also on the boundary conditions. The variation of $n^{*}$ with $\alpha$ can be understood from the competition between the energy cost $\sim N C_{\mathrm{b}} u^{2}$ of purely counter-rotating deformations, and the energy cost $\sim C_{\mathrm{s}} / N u^{2}$ of a shear-induced gradient of these rotations. Balancing these terms yields a characteristic length $n^{*} \sim \sqrt{C_{\mathrm{s}} / C_{\mathrm{b}}} \sim \sqrt{\alpha}$, consistent with our data (Fig. 3a inset). We note that exactly solving the underlying equations of the dressed mechanism in the real space as well as solving the band spectrum in the Fourier space make this argument rigorous, and allow us to formally demonstrate how $n^{*}$ appears as an $\alpha$-dependent bulk quantity, which, surprisingly, is smaller and scales differently than the decay of edge states (see Supplementary Information).

In contrast, the length scale $n_{\mathrm{p}}$ depends on both $\beta$ and the boundary conditions. The variation of $n_{\mathrm{p}}$ can be rationalized through scaling arguments (see Supplementary Information); $n_{\mathrm{p}} \sim \sqrt{\alpha}$ for $\alpha / \beta \ll 1$, whereas $n_{\mathrm{p}} \sim \sqrt{\beta}$ for $\alpha / \beta \gg 1$ : this underlies the data collapse of $n_{\mathrm{p}} \sim \beta^{1 / 2}$ when plotted as function of $(\alpha / \beta)$ (see Fig. $3 \mathrm{~b}$ inset). To probe the boundary dependence of $n_{\mathrm{p}}$, we consider boundary conditions where we independently control the forces $F$ (red) and $F^{\prime}$ (blue) at alternating locations at the edge of the chain, by setting $F^{\prime}=\lambda F$ (Fig. $4 \mathrm{a}$ ). So far, we considered $\lambda=0$, which corresponds to a very sharp indenter, whereas $\lambda=1$ describes a blunt indenter. The intrinsic length scale $n^{*}$ is insensitive to the choice of boundary conditions, but the boundary hybridization factor $\lambda$ allows us to control $n_{\mathrm{p}}$ over a wide range (Fig. $4 \mathrm{~b}$ ): the boundary conditions select the relative strength 
a
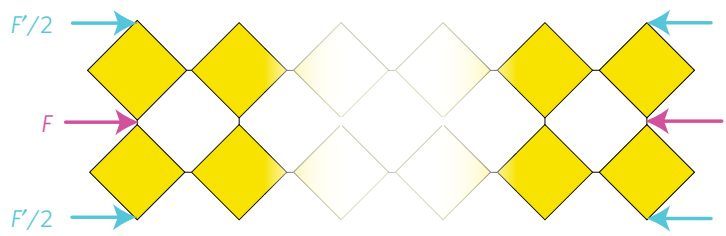

d

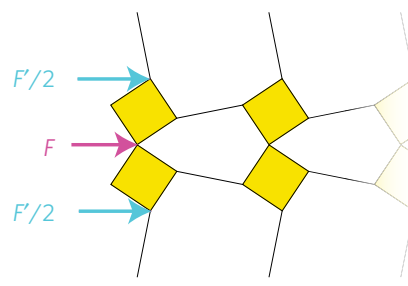

b

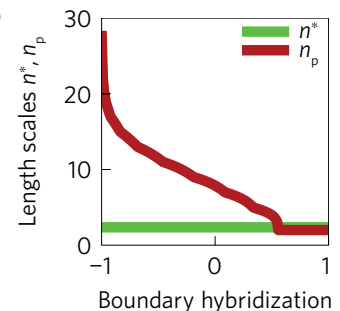

e

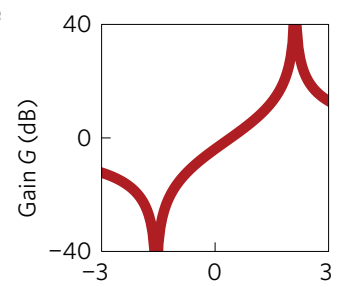

c

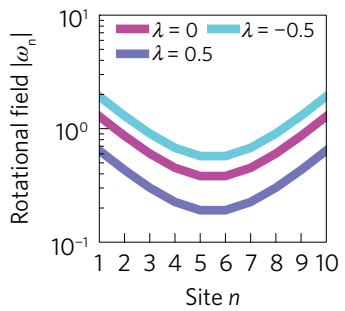

f

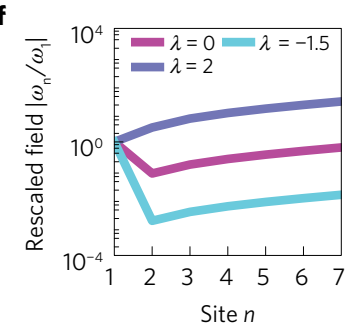

Figure 4 | Sensitivity to boundary conditions. a, Meta-chain with imposed forces $F$ (violet) and $F^{\prime} / 2=(\lambda / 2) F$ (blue). b, Length scales versus $\lambda$, illustrating that $n^{*}$ is an intrinsic feature whereas $n_{p}$ can be tuned by the boundary conditions. $\mathbf{c}$, The amounts of rotation depend strongly on the boundary conditions. Panels b,c were produced using $\alpha=3.38 \times 10^{1}$ and $\beta=1.39 \times 10^{2}$, corresponding to the experimental values. d, Topological chain (see Supplementary Information for the theoretical description). e, The displacement amplification gain $G$ depends strongly on the boundary condition hybridization factor $\lambda$. The gain is defined as $G=20 \log _{10} \omega_{\mathrm{N}} / \omega_{1}$, where $\omega_{1}\left(\omega_{\mathrm{N}}\right)$ is the rotation of the leftmost (rightmost) squares. f, Rotational field $\omega_{n}$ as a function of $\lambda$.

of the mechanism-like rotational deformations and the elastic distortions (Fig. 4c).

To illustrate that this sensitivity to boundary conditions is relevant for a wide class of mechanism-based metamaterials, we consider a topological metamaterial which exhibits one-way motion amplification $^{11}$ (Fig. 4d). In the mechanism limit, such metamaterial is isostatic. Whereas the infinite-bulk-medium is rigid, a finite sample admits one unique zero mode that is either localized on the left or the right, depending on a topological integer-the winding number ${ }^{11-15}$. When mechanically loaded from its hard (soft) side, the metamaterial exhibits amplification (decay). For a hybrid mechanism where the hinges are dressed with torsional and stretch interactions, the boundary conditions control the hybridization of mechanism-like and ordinary elastic deformations. Surprisingly, whereas in the mechanism limit deformations are located near the right boundary, so that forces/displacements excited from the left are amplified, manipulation of the boundary conditions allows us to tune the gain of the displacement amplification over a giant$80 \mathrm{~dB}$-range (Fig. 4e) and to excite deformations that can be localized near the left edge, near the right edge, or near both boundaries (Fig. 4f). Hence, the introduction of finite energy distortions alleviates topological protection and allows boundary programmability.

A physically appealing picture appears: mechanism-based metamaterials have an intrinsic length scale $n^{*}$ that depends on the geometric design and diverges in the purely mechanism limit. Such a length scale quantifies the spatial extension of a soft mode, which localizes near inhomogeneities such as boundaries. Whether this mode is excited depends on the boundary conditions. For the case of the meta-chain, if we choose boundary conditions which are compatible with the counter-rotating texture of the underlying mechanism (that is, $\lambda=-1$ ), the crossover length $n_{\mathrm{p}}$ between mechanism-like and elastic behaviour diverges, whereas strongly incompatible boundary conditions lead to a rapid crossover to ordinary elastic behaviour.

We expect that most mechanism-based metamaterials, including cellular metamaterials $s^{7,9-11,18,23,24}$, allosteric networks ${ }^{28,29}$, gearbased metamaterials ${ }^{15}$ and origami ${ }^{4,16,19-22}$, feature similarly large characteristic scales. Continuum descriptions need to encompass such large scales-in contrast to Cosserat-type descriptions of random cellular solids governed by the bare cell size-as well as the compatibility between the textures of the mechanism and the boundary ${ }^{18}$. We stress that proper hinge design is critical for maintaining functionality in large metamaterial samples, and we suggest exploring hierarchical designs, with multiple small sub-blocks connected via 'meta connectors' that promote the propagation of the required mechanism in each block, thus ensuring that the functionality survives elastic hybridization in the large-scale limit.

\section{Methods}

Methods, including statements of data availability and any associated accession codes and references, are available in the online version of this paper.

Received 8 May 2017; accepted 18 August 2017; published online 25 September 2017

\section{References}

1. Lakes, R. Foam structures with a negative Poisson's ratio. Science 235, 1038-1040 (1987).

2. Grima, J. N. \& Evans, K. E. Auxetic behavior from rotating squares. J. Mater. Sci. Lett. 19, 1563-1565 (2000).

3. Kadic, M., Bückmann, T., Stenger, N., Thiel, M. \& Wegener, M. On the practicability of pentamode mechanical metamaterials. Appl. Phys. Lett. 100, 191901 (2012)

4. Wei, Z. Y., Guo, Z. V., Dudte, L., Liang, H. Y. \& Mahadevan, L. Geometric mechanics of periodic pleated origami. Phys. Rev. Lett. 110, 215501 (2013).

5. Bückmann, T. et al. On three-dimensional dilational elastic metamaterials. New J. Phys. 16, 033032 (2014).

6. Bückmann, T., Thiel, M., Kadic, M., Schittny, R. \& Wegener, M. An elasto-mechanical unfeelability cloak made of pentamode metamaterials. Nat. Commun. 5, 4130 (2014).

7. Mullin, T., Deschanel, S., Bertoldi, K. \& Boyce, M. C. Pattern transformation triggered by deformation. Phys. Rev. Lett. 99, 084301 (2007).

8. Nicolaou, Z. G. \& Motter, A. E. Mechanical metamaterials with negative compressibility transitions. Nat. Mater. 11, 608-613 (2012).

9. Shim, J., Perdigou, C., Chen, E. R., Bertoldi, K. \& Reis, P. M. Buckling-induced encapsulation of structured elastic shells under pressure. Proc. Natl Acad. Sci. USA 109, 5978-5983 (2012).

10. Coulais, C., Overvelde, J. T. B., Lubbers, L. A., Bertoldi, K. \& van Hecke, M. Discontinuous buckling of wide beams and metabeams. Phys. Rev. Lett. 115, 044301 (2015).

11. Coulais, C., Sounas, D. \& Alù, A. Static non-reciprocity in mechanical metamaterials. Nature 542, 461-464 (2017). 
12. Kane, C. L. \& Lubensky, T. C. Topological boundary modes in isostatic lattices. Nat. Phys. 10, 39-45 (2014).

13. Chen, B. G., Upadhyaya, N. \& Vitelli, V. Nonlinear conduction via solitons in a topological mechanical insulator. Proc. Natl Acad. Sci. USA 111, 13004-13009 (2014).

14. Huber, S. D. Topological mechanics. Nat. Phys. 12, 621-623 (2016).

15. Meeussen, A. S., Paulose, J. \& Vitelli, V. Geared topological metamaterials with tunable mechanical stability. Phys. Rev. X 6, 041029 (2016).

16. Waitukaitis, S., Menaut, R., Chen, B. G. \& van Hecke, M. Origami multistability: from single vertices to metasheets. Phys. Rev. Lett. 114, 055503 (2015).

17. Silverberg, J. L. et al. Origami structures with a critical transition to bistability arising from hidden degrees of freedom. Nat. Mater. 14, 389-393 (2015).

18. Coulais, C., Teomy, E., de Reus, K., Shokef, Y. \& van Hecke, M. Combinatorial design of textured mechanical metamaterials. Nature 535, 529-531 (2016).

19. Dudte, L. H., Vouga, E., Tachi, T. \& Mahadevan, L. Programming curvature using origami tessellations. Nat. Mater. 15, 583-588 (2016).

20. Overvelde, J. T. et al. A three-dimensional actuated origami-inspired transformable metamaterial with multiple degrees of freedom. Nat. Commun. 7, 10929 (2016)

21. Overvelde, J. T., Weaver, J. C., Hoberman, C. \& Bertoldi, K. Rational design of reconfigurable prismatic architected materials. Nature 541, 347-352 (2017).

22. Silverberg, J. L. et al. Using origami design principles to fold reprogrammable mechanical metamaterials. Science 345, 647-650 (2014)

23. Florijn, B., Coulais, C. \& van Hecke, M. Programmable mechanical metamaterials. Phys. Rev. Lett. 113, 175503 (2014)

24. Bertoldi, K., Reis, P. M., Willshaw, S. \& Mullin, T. Negative Poisson's ratio behavior induced by an elastic instability. Adv. Mater. 22, 361-366 (2010)

25. Milton, G. W. \& Cherkaev, A. V. Which elasticity tensors are realizable? J. Eng. Mater. Technol. 117, 483-493 (1995).
26. Lechenault, F., Thiria, B. \& Adda-Bedia, M. Mechanical response of a creased sheet. Phys. Rev. Lett. 112, 244301 (2014).

27. Landau, L. D. \& Lifshitz, E. M. Theory of Elasticity (Pergamon, 1970).

28. Rocks, J. W. et al. Designing allostery-inspired response in mechanical networks. Proc. Natl Acad. Sci. USA 114, 2520-2525 (2017).

29. Yan, L., Ravasio, R., Brito, C. \& Wyart, M. Architecture and coevolution of allosteric materials. Proc. Natl Acad. Sci. USA 114, 2526-2531 (2017).

\section{Acknowledgements}

We thank J. Mesman for outstanding technical support and A. Alù, P. Dieleman, E. Lerner, A. Meeussen, and A. Souslov for insightful discussions. We acknowledge funding from the Netherlands Organization for Scientific Research through grants VICI No. NWO-680-47-609 (M.v.H.) and VENI NWO-680-47-445 (C.C.).

\section{Author contributions}

C.C. and M.v.H. conceived the project. C.C. and C.K. designed the experiments. C.K. performed the experiments and analysed the data. C.C. carried out the numerical simulations. C.C., C.K. and M.v.H. developed the theoretical models. C.C. and M.v.H wrote the manuscript.

\section{Additional information}

Supplementary information is available in the online version of the paper. Reprints and permissions information is available online at www.nature.com/reprints. Publisher's note: Springer Nature remains neutral with regard to jurisdictional claims in published maps and institutional affiliations. Correspondence and requests for materials should be addressed to C.C.

\section{Competing financial interests}

The authors declare no competing financial interests. 


\section{Methods}

Experiments. We fabricate our samples by $3 \mathrm{D}$ printing a flexible polyethylene/polyurethane thermoplastic mixture (Filaflex by Recreus, Young's modulus $E=12.75 \mathrm{MPa}$, Poisson's ratio $v \sim 0.5$ ). The samples are $7.5 \mathrm{~mm}$ thick, initially made of $N=14$ rows of squares of diagonal $L=17 \mathrm{~mm}$, which are connected by ligaments of length $\ell=1.7 \mathrm{~mm}$ and width $w=1.7 \mathrm{~mm}$ (Fig. 2a,b of the main text). We measure the stiffness of these samples by pinching the outer horizontal joints between two rods, which are positioned such that they tightly grip the joints-this boundary condition ensures that the rotational mode is strongly excited. The rods are attached to an uniaxial testing device equipped with a $100 \mathrm{~N}$ load cell, which measures compressive forces $F$ and compressive displacements $\delta$ with $1 \mathrm{mN}$ and $10 \mu \mathrm{m}$ accuracy, respectively, and with which we apply an external displacement from $\delta=0.50 \mathrm{~mm}$ (in compression) to $\delta=-1.50 \mathrm{~mm}$ (in extension). We focus on the linear response regime, and measure the stiffness $k$ in the displacement range $\delta \in[0.25 \mathrm{~mm},-1.25 \mathrm{~mm}]$ by using the linear coefficient of a second-order polynomial fit to the force-displacement $F$ versus $\delta$ curve (see Supplementary Fig. 1a), leading to a relative error ( $95 \%$ margin of confidence) of less than $0.2 \%$ for the value of the stiffness. Note that it is important to include the quadratic correction in the fitting procedure to obtain an accuracy below $1 \%$. To measure the variation of $k$ with $N$, we print $N=14$ samples, perform experiments, remove a pair of squares to obtain $N=13$, perform more experiment and so on.

We have marked these elements and record images with a high-resolution complementary metal-oxide-semiconductor (CMOS) camera (Basler acA2040-25gm; resolution $4 \mathrm{Mpx}$ ), which is triggered by the mechanical testing device. This allows us to measure rotations $\theta(n)$ with $1 \times 10^{-1}$ deg accuracy versus the displacement $\delta$. In the linear regime, $\theta(n)$ is proportional to $\delta$, and we determine the rotational rate $\omega(n)$ from a linear fit of the $\theta(n)$ versus $\delta$ curves (see Supplementary Fig. 1b).

Numerical simulations. For our static finite elements simulations, we use the commercial software Abaqus/Standard and a neo-Hookean energy density as a material model, with a shear modulus, $G=4.25 \mathrm{MPa}$ and bulk modulus, $K=212 \mathrm{GPa}$ (or equivalently a Young's modulus $E=12.75 \mathrm{MPa}$ and Poisson's ratio $v=0.49999$ ) in plane strain conditions with hybrid quadratic triangular elements (abaqus type $\mathrm{CPE} 6 \mathrm{H}$ ). We perform a mesh refinement study to ensure that the thinnest parts of the samples where most of the stress and strain localized are meshed with at least four elements. As a result, the metamaterial has approximately from $3 \times 10^{3}$ to $6 \times 10^{4}$ triangular elements, depending of the value of $N$.

Simulation of the full metamaterial. We simulate the full metamaterial by applying boundary conditions by pinching the most outer vertical connections as in the experiments. We impose a small displacement of magnitude $\delta=3 \times 10^{-4} L$ to the structure and measure the reaction force $F$. Given that such small displacement ensures the structure is probed in its linear response, we estimate the stiffness as $k=F / \delta$.

Measurement of the hinge stiffnesses. The shear, bending and stretching stiffnesses all depend strongly on the hinge geometry, including the width $w$. In simple cases such as hinges of constant cross-section, it is possible in the slender limit $(w \ll \ell)$ to use beam theory to give analytical expressions for these stiffnesses versus the hinge geometry and the bulk material elastic constants. However, our hinges are neither in the slender-beam limit nor of constant cross-section (the squares also deform and as such are part of the hinges, as can be readily seen in Supplementary Fig. 4a). Therefore we estimate them using FEM, presented in the following.

We measure the individual bending, stretch and shear stiffness by simulating two squares connected by one elastic ligament and applying three sorts of boundary conditions depicted in Supplementary Fig. 3. To apply bending, stretching and shear boundary conditions, we define constraints for every node on the vertical diagonal of each square and assign their displacements to the motion of a virtual node, which is then displaced by a small amount $\delta=3 \times 10^{-4} L$. We then extract the reaction forces $F_{\mathrm{b}}, F_{\mathrm{j}}$ and $F_{\mathrm{s}}$, respectively on this virtual node to calculate the stiffnesses as follows

$$
\begin{gathered}
C_{\mathrm{b}}=\frac{L^{2}}{4} \frac{F_{\mathrm{b}}}{\delta} \\
k_{\mathrm{j}}=\frac{F_{\mathrm{j}}}{\delta} \\
C_{\mathrm{s}}=\frac{\ell^{2}}{2} \frac{F_{\mathrm{s}}}{\delta}
\end{gathered}
$$

Data availability. The data that support the plots within this paper and other findings of this study are available from the corresponding author upon request. 